\title{
e-Phaïstos
}

e-Phaïstos Revue d'histoire des techniques / Journal of the history of technology

IX-2 | 2021

Quel objet pour quel musée?

\section{L'objet technique}

Au cœur du musée d'Art et d'Industrie de la ville de Saint-Étienne The Technical Object: at the Heart of the Saint-Etienne Museum of Art and Industry

\section{Marie-Caroline Janand}

\section{(2) OpenEdition}

\section{Journals}

Édition électronique

URL : https://journals.openedition.org/ephaistos/9278

DOI : 10.4000/ephaistos.9278

ISSN : 2552-0741

Éditeur

IHMC - Institut d'histoire moderne et contemporaine (UMR 8066)

Référence électronique

Marie-Caroline Janand, "L'objet technique », e-Phaïstos [En ligne], IX-2 | 2021, mis en ligne le 26 octobre 2021, consulté le 28 octobre 2021. URL : http://journals.openedition.org/ephaistos/9278 ; DOI : https://doi.org/10.4000/ephaistos.9278

Ce document a été généré automatiquement le 28 octobre 2021.

Tous droits réservés 


\title{
L'objet technique
}

\author{
Au cœur du musée d'Art et d'Industrie de la ville de Saint-Étienne \\ The Technical Object: at the Heart of the Saint-Etienne Museum of Art and \\ Industry
}

Marie-Caroline Janand

1 Le musée d'Art et d'Industrie conserve et valorise le patrimoine représentatif des grandes industries de la région stéphanoise. Le musée porte l'appellation musée de France.

2 Le premier musée municipal est hébergé dans les années 1830 dans l'hôtel de ville. Les collections sont des minéraux, quelques monnaies et des animaux naturalisés. Dans les années 1850, la ville achète la collection d'armes du maréchal Oudinot. Dès ce moment, l'orientation clairement industrielle du musée est posée. Les collections doivent servir les industries du territoire en lien avec l'école de dessin naissante. Les conservateurs sont alors souvent des armuriers, ou des patrons textiles. Marius Vachon (1850-1928), conservateur du musée à la fin du XIX ${ }^{\mathrm{e}}$ siècle, théorise les liens entre le musée et l'industrie suivant les modèles anglo-saxons issus du mouvement Arts and Crafts (Passini 2014). Le musée, qui passe de "Palais des Arts » à " Musée d'Art et d'Industrie », est hébergé depuis 1861 dans un bâtiment dont la destination première était de devenir la sous-préfecture de la Loire.

3 Le musée conserve aujourd'hui près de 45000 objets de typologies variées. L'institution est réputée pour sa collection d'armes, soit 7000 pièces complètes et un grand nombre de pièces détachées, qui en fait la deuxième en France après le musée de l'Armée. Il conserve la première collection de rubans au monde avec plus d'un million d'échantillons, 150 machines textiles, des cours techniques ainsi que des centaines de dessins de Fabrique et mises en cartes. L'ensemble des cycles est le premier de France avec près de 400 machines de toutes les époques et des pièces détachées représentatives de l'industrie stéphanoise. Le fonds d'affiches de cycles, soit 650 pièces, est de première importance, tout comme la documentation collectée autour du deux roues. A côté de ces trois collections, qui sont au cœur de la scénographie actuelle, figurent des ensembles remarquables en réserves comme la numismatique, notamment 
les monnaies et médailles des XVIII ${ }^{\mathrm{e}}$ et XIX ${ }^{\mathrm{e}}$ siècles, les grands noms de la gravure de monnaie en France de 1750 à 1880 étant stéphanois. A cela s'ajoutent le mobilier et l'art décoratif qui, s'ils sont moins cohérents et de qualité inégale, présentent quelques pièces fort rares.

Depuis 2018, la Ville de Saint-Étienne engage un rapprochement du musée d'Art et d'Industrie et de Couriot musée de la Mine, qui fêtera ses 30 ans en 2021. Les deux institutions représentent un pôle d'attraction touristique important avec plus de 100000 visiteurs annuels. Ce rapprochement se traduit en 2021 par un nouveau Projet Scientifique et Culturel, en cours de validation par les tutelles, définissant notamment les axes de collecte, de présentation et de médiation. La réflexion menée dans le cadre de ce projet, autour de ce qui fait sens commun à ces deux institutions aux identités bien différentes, a permis de dégager de très nombreux points de convergence. L'un d'entre eux est la nature des collections, puisque l'objet technique est au cœur des deux musées, avec toutefois une grande différence dans la valeur qui lui est attachée.

Poser la question de l'évolution des valeurs accordées à l'objet technique dans l'histoire d'une institution comme le musée d'Art et d'Industrie de Saint-Étienne, qui plus est dans le contexte d'une évolution patrimoniale exprimée dans un Projet Scientifique et Culturel conçu pour deux musées, apparaît donc légitime. C'est, en creux, tenter de définir la finalité de l'objet technique en musée, dans une approche chronologique depuis les années 1870 à nos jours.

\section{La valeur démonstrative de l'objet technique au musée d'Art et d'Industrie}

6 Le musée naît de la volonté de relancer des arts français dans un contexte général de relèvement de la France après la guerre de 1870. L'affaiblissement des industries d'art est un sentiment répandu, plus qu'une réalité, qui amène l'État à demander à Marius Vachon une série de rapports comparatifs entre la production hexagonale et celle de différents pays (Vachon 1885). C'est sur cette base que Vachon va concevoir pour SaintÉtienne un musée chargé de former l'artisan comme l'ouvrier. Ce musée est au cœur de sa conception de renouvellement de l'art français, lui qui théorise un long déclin de l'art industriel depuis la fin des corporations en 1791. On trouve donc à côté des productions stéphanoises des collections d'œuvres d'art, moins pour la délectation que pour permettre aux ouvriers d'art d'approcher le «bon goût » (fig.1). C'est pourquoi sont exposés alors des peintures, estampes et objets d'art décoratif. Ces fonds sont constitués en parallèle des pièces liées aux industries, l'iconographie renvoyant le plus souvent à ces collections phares : tableaux de fleurs sur le modèles lyonnais ou objets orientaux pour le ruban, scènes de chasse ou fantaisies à la Bérain pour la gravure sur arme... 
Fig.1. Potiche à couvercle en porcelaine d'Arita (Japon - XVIII siècle)

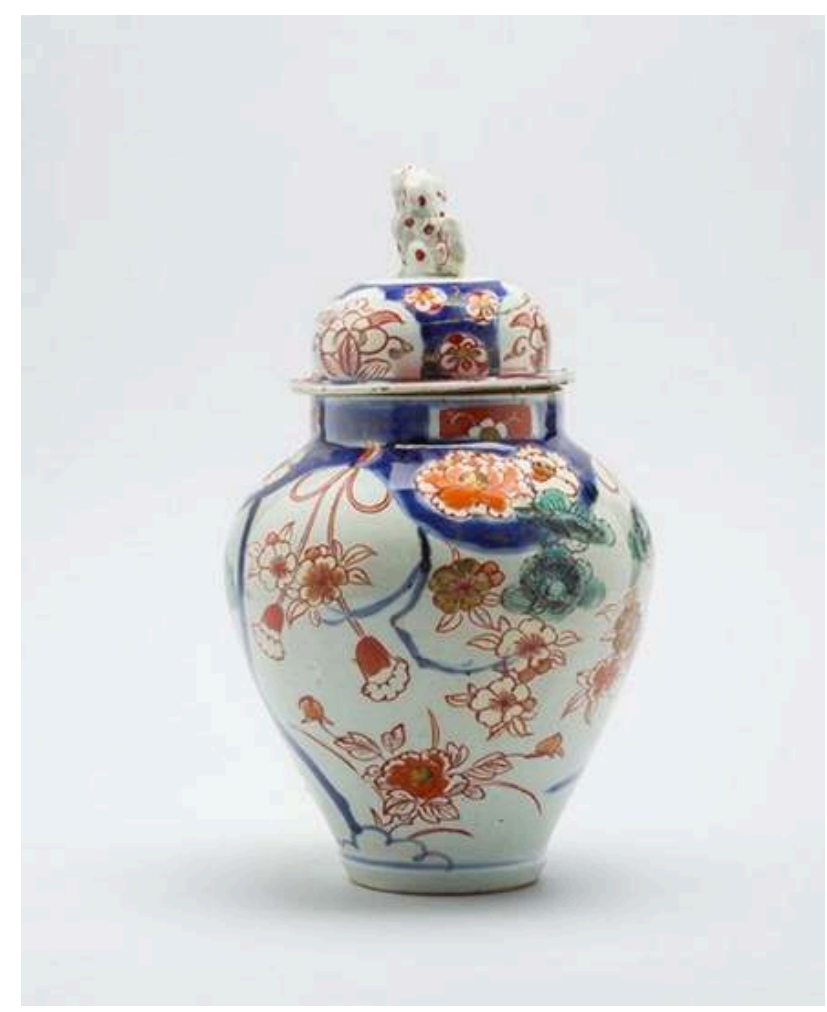

Cette potiche est représentative des pièces collectées pour servir de modèles esthétiques aux dessinateurs de Fabrique. Au XIX ${ }^{e}$ siècle, le Japon est à la fois une source d'inspiration pour des rubans stéphanois destinés au marché européen, mais aussi pour des pièces qui seront exportées en Extrême-Orient.

Musée d'Art et d'Industrie, inv. 2008.0.477

7 Mais ces ensembles, aussi riches soient-ils, ne masquent pas la valeur démonstrative des objets techniques érigés comme modèles bien avant que Vachon ne devienne conservateur de l'institution en 1890 (Besse 2005). La valeur d'usage attaché à l'objet technique est dès le début du musée celle de la démonstration pédagogique, valeur renforcée par le discours de Marius Vachon. Démonstration esthétique, avec des pièces choisies parmi les «plus belles» dans la collection du maréchal Oudinot par le conservateur alors en place, Christophe Jalabert (1799-1876), armurier de son état; mais aussi, et surtout, démonstration technique des savoir-faire autour de la petite métallurgie, puis du textile. C'est ainsi que des pièces d'excellence intègrent les fonds, fusils « chefs-d'œuvre », mais aussi des objets servant d'exemplarité technique comme des coupes d'armes où le mécanisme est mis à nu (fig.2). 
Fig.2. Platine gauche à silex. Pierre Missillieux, Vienne (Autriche), vers 1840

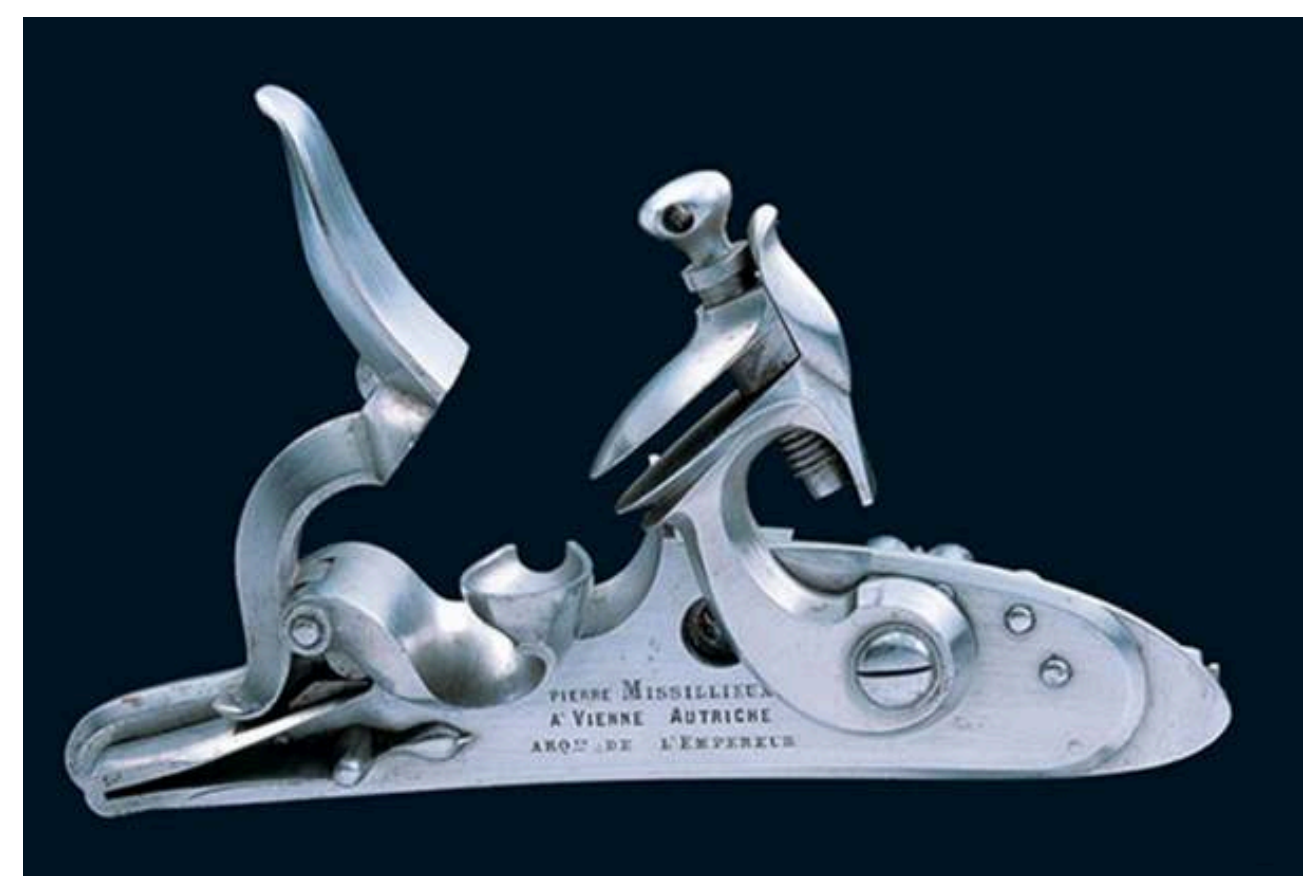

L'exposition de pièces détachées d'armes, comme les platines, participe à la volonté de faire un musée pour servir l'analyse technologique. La formation de l'ouvrier et/ou de l'artisan repose sur l'acquisition des savoir-faire dont fait partie la connaissance des objets techniques présentés au musée.

Musée d'Art et d'Industrie, inv. 2003.19.78

8 C'est dans le même esprit que le musée achète des armes étrangères, notamment liégeoises, lors des expositions universelles, leur démontage/remontage permettant aux armuriers stéphanois d'analyser la force technique de la concurrence. Le musée participe alors à l'espionnage industriel. Dans le domaine textile, le fonds d'échantillons et de modèles constitué vers 1844 est rapidement augmenté par les registres des Prud'hommes déposé par la Chambre de commerce. Ce fait illustre les liens étroits entre l'institution muséale stéphanoise et le monde de l'entreprise: les professionnels voyant le musée comme le lieu de la sauvegarde des dépôts de modèles. La présentation de ces objets reste toutefois très académique, calquée sur un musée de Beaux-arts, en une succession de vitrines procédant par accumulation. Le public est mis à distance, autant par l'éloignement physique des objets techniques que par leur changement de valeur qui les érigent en modèles. La dichotomie est frappante entre la volonté de faire du musée un lieu de savoir et d'accès à l'objet pour l'ouvrier ou l'artisan et la réalité d'une monstration le théâtralisant et l'éloignant des notions de fabrication et d'usage (fig. 3). 
Fig.3. Vue des salles d'armes entre 1930 et 1944

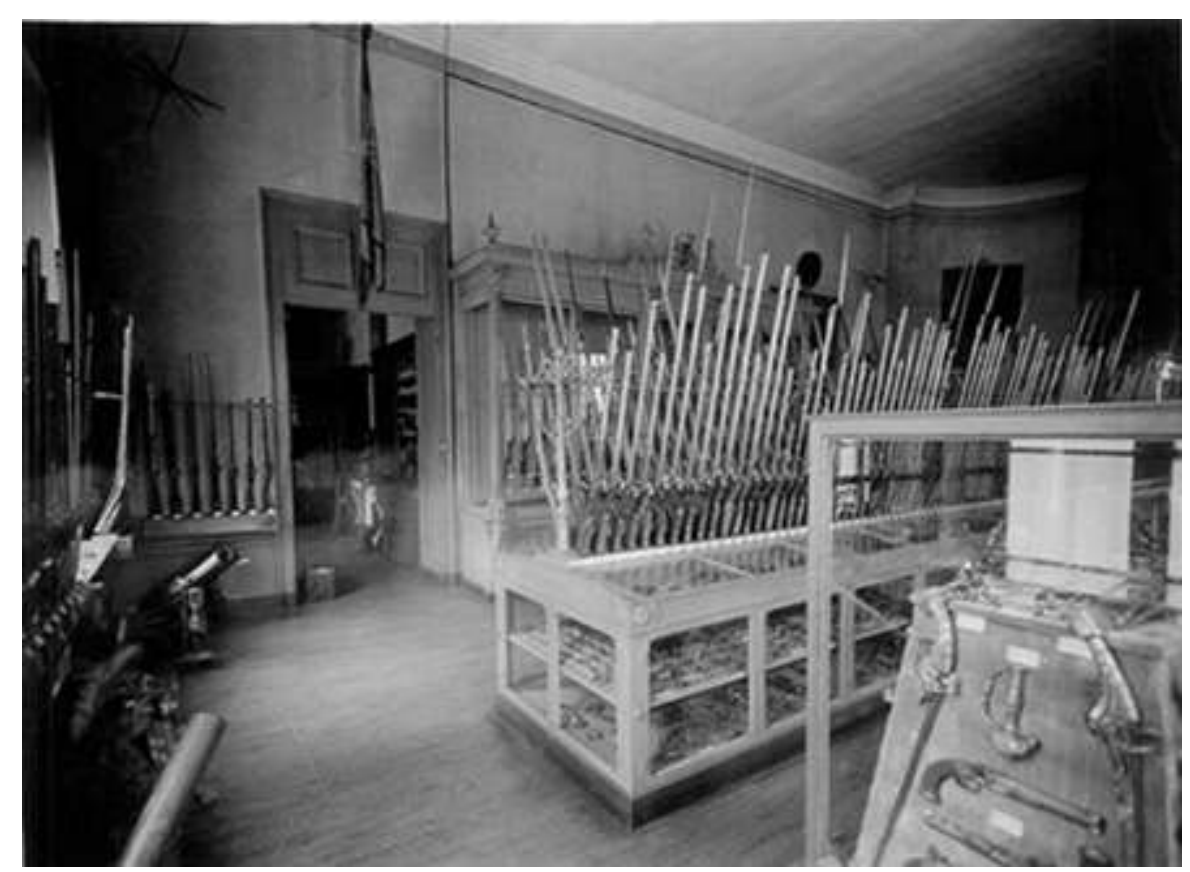

L'objet technique est présenté dans un principe accumulatif typique des musées au XIX`e siècle. La volonté de Marius Vachon est de faire un musée pour former l'armurier sur la technique et l'esthétique de l'arme. Dans les faits, comme le montre cette photographie, la densité de la présentation ne permet ni analyse ni comparaison.

Documentation histoire de l'institution - musée d'Art et d'Industrie

9 La structure des collections du musée de Saint-Étienne est profondément modifiée sous l'impulsion de Maurice Allemand, conservateur de 1947 à 1966. Grand connaisseur d'art moderne, très introduit dans les milieux artistiques, il va multiplier les acquisitions d'œuvres contemporaines et les montrer, soit lors d'expositions temporaires qui font date, soit dans les collections permanentes (Bargues 2021). L'ensemble de ces pièces constitue le noyau des collections du musée d'art moderne et contemporain de SaintÉtienne. Maurice Allemand n'entre toutefois pas en rupture avec l'objet technique. C'est lui qui va impulser la constitution d'une collection de cycles anciens et d'affiches de premier ordre, faisant ainsi entrer au musée une autre production stéphanoise. L'achat d'armes africaines ou de statuettes de tisserands est mentionné dans les inventaires pour servir les "collections techniques". Les pièces sont exposées en vitrines, mais choisies avec soin, pour montrer une filiation entre l'objet technique et les collections artistiques (fig. 4). La valeur démonstrative est toujours présente mais glissant pour certains objets vers une présentation esthétique dans un continuum d'histoire de l'art. 
Fig.4. Vue d'une salle mêlant objets techniques et art moderne en 1949

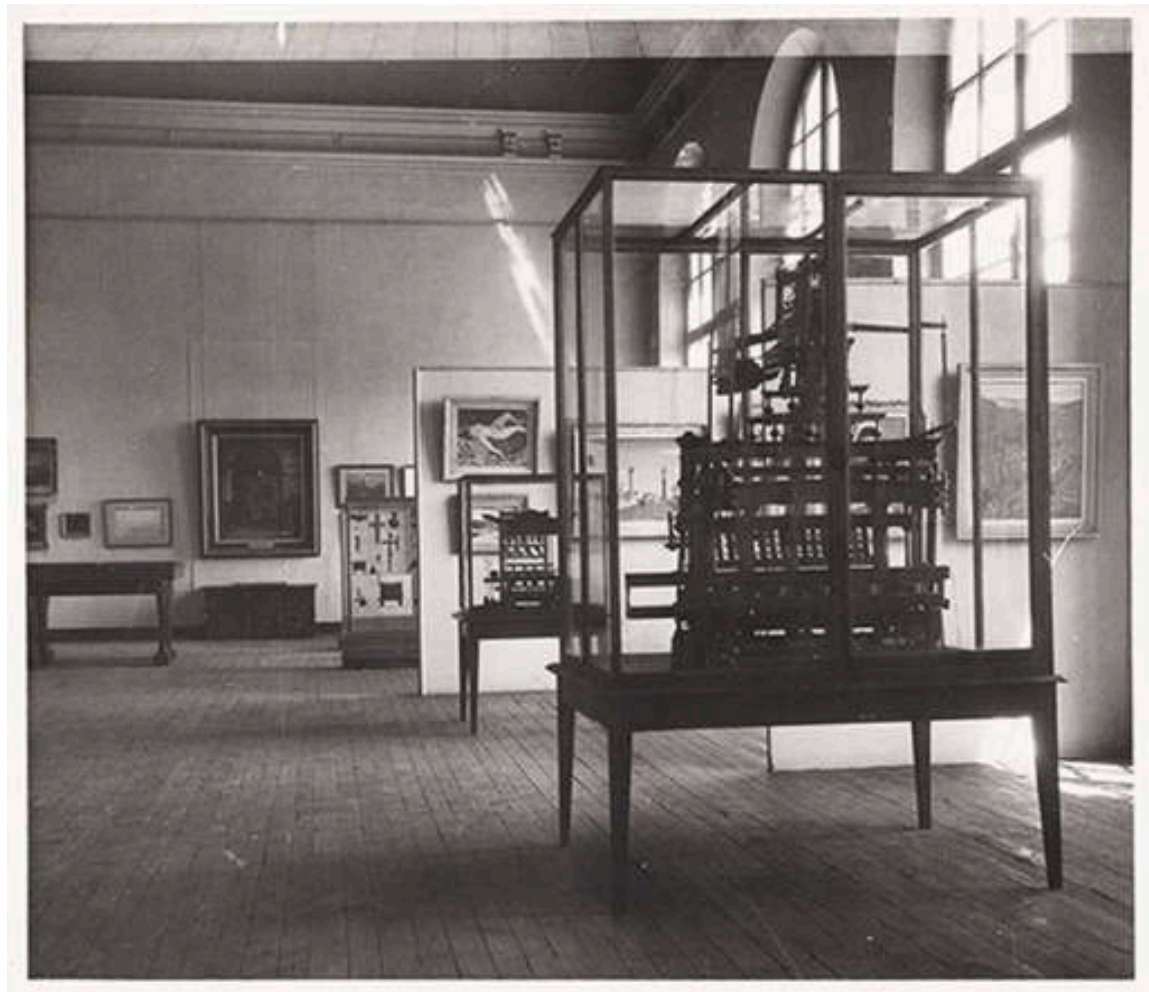

Maurice Allemand modifie les salles très tôt après sa prise de poste en 1947. II fait dialoguer les objets techniques; ici des maquettes de métiers à tisser, et l'art moderne et ancien. On reconnaît notamment un nu de Jean Puy et, plus au fond, une série d'émaux médiévaux.

Documentation histoire de l'institution - musée d'Art et d'Industrie

\section{De la valeur didactique à la valeur d'expert}

L'ouverture du musée d'art moderne en 1988 et le départ des collections d'art ancien vers cette institution font évoluer le rapport à l'objet technique. La valeur de celui-ci mute lors de la rénovation de 2001 dans laquelle Jean-Michel Wilmotte met en œuvre le redéploiement, sur plus de $2500 \mathrm{~m}^{2}$, de trois collections internationalement reconnues : le ruban, l'arme et le cycle, s'appuyant sur un document rédigé en 1993 par le conservateur Nadine Besse. Dans cette muséographie, la valeur démonstrative s'efface devant une approche historique de la production, en lien avec les évolutions techniques. Les liens avec l'art, et la valeur didactique qui en découlait, sont moins présents. La présentation est sobre et élégante, l'objet technique devenant une véritable œuvre d'art sur des podiums ou dans des vitrines avec peu, voire pas, d'éléments de contexte. La richesse des collections est matérialisée par l'abondance des pièces, notamment pour les cycles et les armes. L'objet technique devient le support d'un parcours d'expert, accompagné de vidéos où sont décortiqués les mécanismes. Des mises en situation d'ateliers d'armurier ponctuent le parcours au niveau des armes mais il y a peu d'explications sur la manière dont les fusils sont fabriqués et aucune sur leur usage. La même remarque peut être faite dans les salles cycles. La place du médiateur est essentielle pour saisir tant la portée technique, que la révolution sociale ou économique des objets et seule la visite guidée permet au public de saisir les spécificités techniques des pièces présentées. La répartition des typologies de 
collections par niveau accentue la dissociation des objets et ne permet pas au visiteur individuel d'appréhender les porosités technologiques.

Les métiers à tisser qui fonctionnent, animés par une équipe de passementiers bénévoles et un médiateur-gareur, occupent une place à part dans ce parcours permanent. Le public est toujours placé comme observateur mais immergé dans la fabrication. Le "sachant» représenté par le médiateur s'efface devant le "faiseurraconteur ", détenteur d'un savoir de terrain qui apparait, à tort ou à raison, comme plus véridique dans un discours moins codifié (fig.5). En sortie de visite, le public se souvient moins des rubans que de l'échange avec les passementiers. C'est bien cet échange qui est au cœur de la relation à l'objet technique, ce n'est ni le métier à tisser, ni l'objet fini, le ruban. Le visiteur vit une expérience, la distance avec l'objet est abolie, il est même invité à passer près du passementier, en situation de « faiseur ». Auprès des passementiers, la perte d'identité de l'objet technique, inhérente au processus muséal mais fortement accentuée par une muséographie faite pour des experts, est comblée par l'expérience avec le «faiseur-raconteur». La réalité rétive décrite par Philippe Mairot (Mairot 1991), celle des gestes et des savoir-faire si difficiles à montrer en musée mais qui est essentielle pour appréhender l'ensemble des concepts liés aux objets techniques, est synthétisée dans la figure du passementier.

Fig.5. Présentation des métiers en fonctionnement

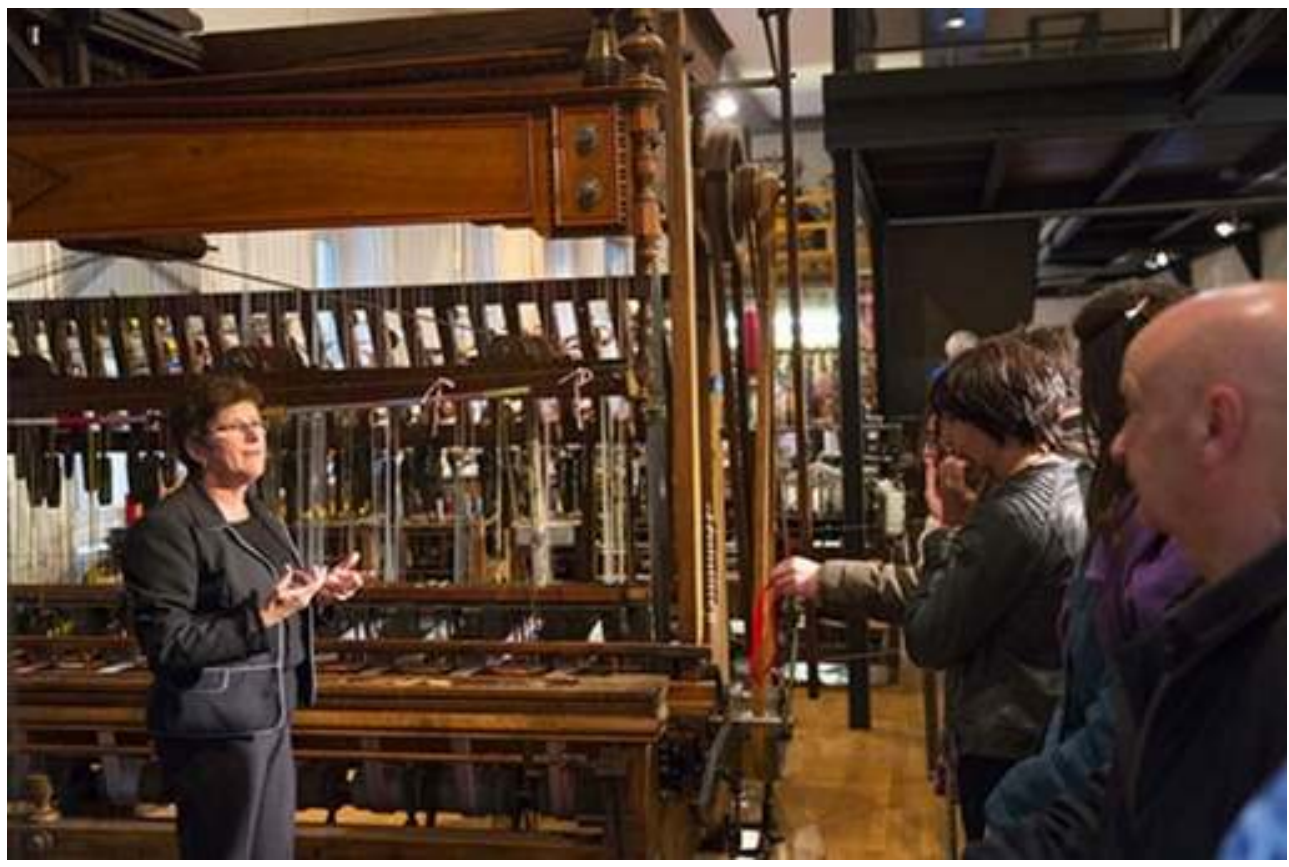

La présentation est faite ici par un gareur-médiateur. Ce métier est spécifique aux musées textiles, le gareur étant la personne qui monte les fils sur la machine selon la production souhaitée et qui va s'assurer du bon déroulement de la fabrication. Le gareur-médiateur au musée d'Art et d'Industrie est une femme, technicienne textile qui a travaillé en production. Elle anime aussi le réseau des passementiers bénévoles qui viennent s'occuper des machines et faire des démonstrations. Le renouvellement de cette équipe de bénévoles est un enjeu pour le musée.

Documentation histoire de l'institution - musée d'Art et d'Industrie 


\section{La valeur contextuelle de l'objet technique}

12 La collection du musée de Saint-Étienne est donc non seulement composée d'objets techniques mais destinée à servir la fabrication de l'objet technique. Cette mise en abîme est progressivement abandonnée avec le déclin de deux des industries phares du territoire, le cycle et l'arme, dès la fin des années 1980. La structuration de l'enseignement technologique, qui se réoriente vers des cursus plus généralistes de mécanique ainsi que l'accès à un savoir plus universel en un clic, et donc à un corpus de modèles techniques et esthétiques infini, modifient profondément le statut de l'objet dans un musée dont la muséographie est destinée à l'expert. Le musée d'Art et d'Industrie de 2001 met l'accent sur trois collections dont la production était encore présente sur le bassin stéphanois lors de la rédaction du projet. Toutefois, le tissu économique a profondément muté depuis. La production de cycles a totalement disparu. Même si on observe toujours une présence de l'armurerie liée aux savoir-faire historiquement mis en œuvre dans cette production, au banc d'épreuve qui est resté à Saint-Étienne et à la formation d'excellence assurée dans un lycée professionnel stéphanois, il reste que la fabrication d'armes se cantonne à une entreprise et quelques ateliers. Seule la production de ruban est encore très présente sur le bassin avec, pour certaines entreprises, une mutation vers le textile technologique et/ou médical. Cette production est toutefois bien éloignée de la restitution faite au musée de l'Atelier familial du passementier, avec aujourd'hui une forte mécanisation et la nécessité de rendements importants.

La rupture entre le musée d'Art et d'Industrie et le public est donc profonde. Alors que l'activité minière a cessé sur Saint-Étienne en 1973, soit une vingtaine d'années avant la fin de l'industrie du cycle, le ressenti est très différent au musée de la Mine. La présence du site, qu'on peut presque considérer comme un objet technique à part entière, est si forte et la figure du mineur si souvent mythifiée que le public ne ressent pas de rupture. En effet, le public se perçoit comme un élément de l'objet technique, il descend vivre l'expérience du fond et le gigantisme des machines en surface l'absorbe. C'est ainsi qu'au musée de la Mine, paradoxalement, la collection est peu importante en nombre d'objets avec quelque 3500 items et, s'il se trouvent des objets techniques (marteaux piqueurs, engins de sauvetage, lampes...), il y aussi un nombre important de pièces graphiques. La valeur accordée à l'objet technique à Couriot musée de la Mine est mémorielle, et pose in-fine bien moins de questions que l'objet technique au musée d'Art et d'Industrie. L'objet est là pour témoigner du passé et le site, comme méga-objet technique, rempli cet objectif à lui tout seul. L'attention portée jusqu'ici à la collection par les conservateurs successifs a ainsi toujours été moindre que celle accordée au site.

$\mathrm{Au}$ contraire de cette immersion physique qui peut seule suffire à instaurer un lien émotionnel, la visite au musée d'Art et d'Industrie nécessite de la part du public de très nombreux prérequis techniques, historiques et sociaux (fig. 6). Les liens se sont distendus, qu'il s'agisse du lien de production comme du lien d'usage, et la plupart des visiteurs ne sait pas pourquoi les objets sont présentés sur ce territoire, qui plus est dans une muséographique dénuée d'information contextuelle qui maintient la distance usager/objet technique telle que décrite par Patrice Flichy (Flichy 2008). L'enjeu d'une nouvelle forme de présentation de l'objet technique au musée d'Art et d'Industrie consiste à faire coïncider deux univers de référence, celui du visiteur et celui de l'objet. Pour cela la muséographie doit être le lieu de l'organisation d'un savoir dans lequel se 
place l'objet technique et qui le dépasse, permettant d'accéder au contexte global car " on ne peut guère [..] appréhender un fait technique qui ne soit aussi, dans le même temps, un fait social, symbolique et économique et que l'effort de l'ethnologie consiste à mettre en lumière cette totalité dynamique » (Mairot 1991).

Fig.6. Vue des salles de cycles en 2019

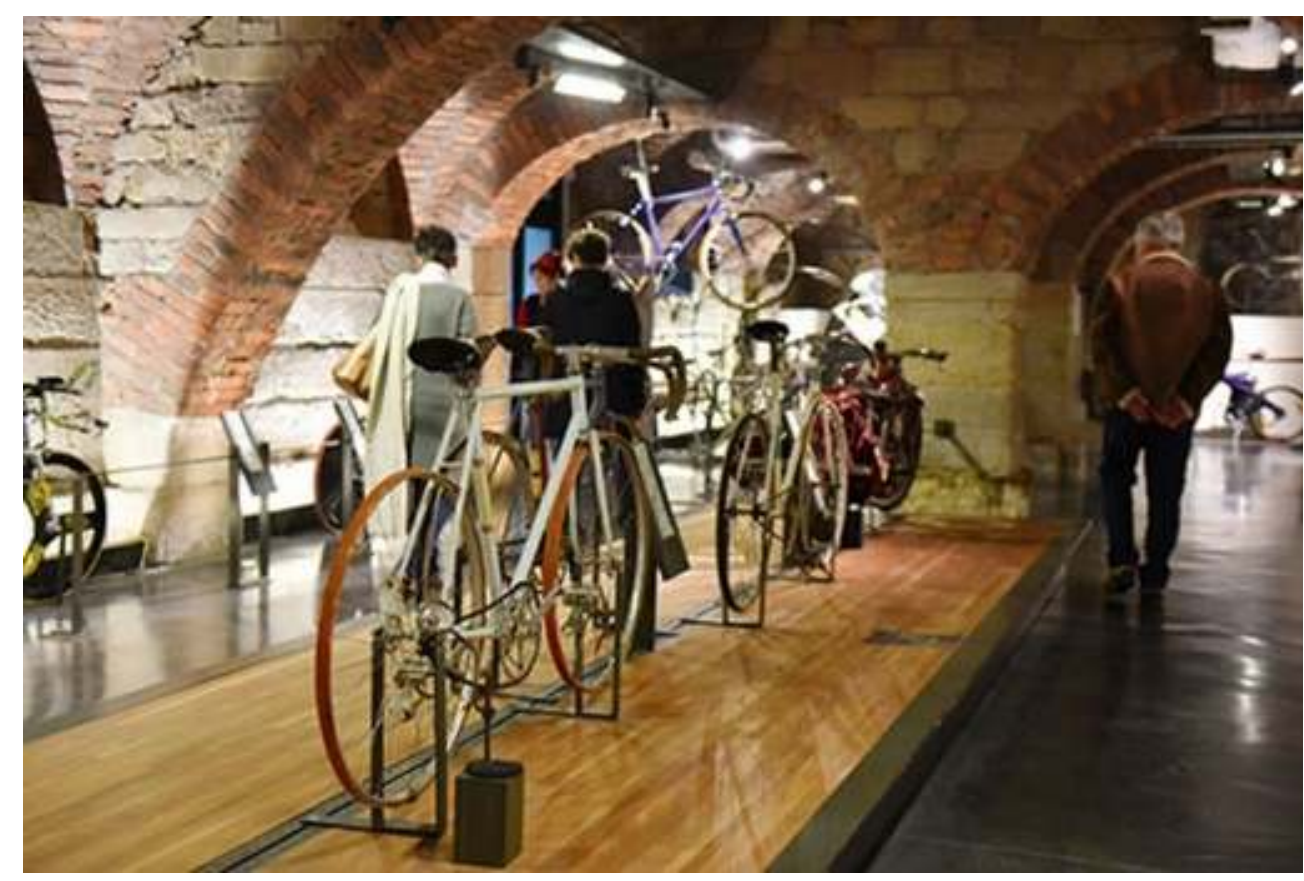

La vue montre l'élégant podium dédié à la piste. Une motocyclette, qui se distingue en rouge, « lançait » les coureurs dans les vélodromes. Les cartels s'intercalent avec les objets techniques mais il n'y a aucune pièce contextuelle permettant de relier par l'iconographie la production sur le territoire de motocyclettes, le vélodrome qui existait à Saint-Étienne et les spécificités techniques des machines présentées.

Ville de Saint-Étienne-Charlotte Pi

Il faut donc reconnecter le public à l'objet et, plus globalement, au musée. La contextualisation, qui n'est pas la reconstitution, est un des outils de cette reconnexion en organisant le savoir dans lequel se place l'objet. Car la reconstitution d'un atelier d'armurier ne suffit pas à faire comprendre la fabrication d'une arme, comme l'exposition d'une robe haute couture ne permet pas d'envisager tous les usages du ruban. Il ne s'agit pas de livrer l'ensemble du contexte autour de l'objet technique par un foisonnement d'archives, de photographies et de témoignages, mais de recomposer le savoir dans une appréciation plus transversale qui ne se concentre pas uniquement sur la technologie, l'histoire, la production ou l'usage. Le nouveau projet veut mettre en résonance les objets entre eux, retrouver les liens avec l'art et, plus globalement, proposer des thématiques transversales autour d'objets polysémiques. Ces objets sont des clés pour appréhender l'histoire du territoire, le mode de production, les savoirfaire, les innovations techniques, les acteurs de sa diffusion (marchés, commerce...), l'organisation sociale... C'est ainsi que des espaces transversaux donneront des clés de compréhension de l'objet technique. Seront abordés par exemple l'importance de la mécanique comme lien entre les productions ou le rôle du musée dans la diffusion des modèles. Chaque temps de transversalité consiste en un objet principal (objet 
polysémique) et des objets/documents satellites avec pour chaque espace une médiation.

16 Le public a profondément évolué en vingt ans. Cette véritable mutation sociale est la conséquence de plusieurs facteurs comme la porosité des temps sociaux (travail famille loisirs), l'empreinte profonde du numérique ou la facilité de déplacement des individus. La diffusion de l'image de la culture est internationale et l'immense majorité des individus a, dans les pays européens, un accès immédiat au savoir. Le frein à la diffusion du savoir est moins une question d'accès et de coût du matériel que l'éducation à la recherche afin d'opérer un tri dans ces savoirs. Les dernières études montrent que le musée reste identifié comme le lieu d'un savoir "sûr ». Cette mutation sociétale impacte donc l'individu. Plus connecté, il fractionne ses pratiques culturelles et sa manière d'appréhender les savoirs, comme il fractionne sa consommation numérique en zappant d'un contenu à l'autre. Le corollaire est une attention moins grande, surtout pour regarder un objet «fixe» comme ceux qu'on montre dans les musées. L'accès démultiplié au savoir s'accompagne paradoxalement d'un amoindrissement des connaissances dans les prérequis attendus pour la visite, notamment en matière de chronologie et connaissances historiques.

La scénographie est un moyen de reconnecter le public au musée et à l'objet technique. Dans ce contexte, le numérique peut être vu comme un outil très intéressant d'approche de la technologie de l'objet technique, en développant par exemple le mécanisme de fonctionnement dans une proposition virtuelle. Mais le numérique n'est pas le seul outil, l'expérimentation du processus physique reste un facteur essentiel de la médiation autour de l'objet technique. Car une des particularités de ces objets demeure leur fonctionnement. Le public souhaite comprendre comment l'objet entre en mouvement, ce qui est expliqué pour certains mécanismes dans les vidéos pédagogiques développées dès 2001 et qui demandent à être réactualisées au vu des nouveaux moyens que permet le numérique. C'est l'analyse technologique de l'objet. Mais la demande du public dépasse largement ce cadre, et il attend du musée le lieu d'un savoir global, notamment les relations de l'objet et de l'homme : c'est l'analyse d'usage (fig. 7). Cette notion recouvre notamment la relation sociale à l'objet, ses permanences (le retour du cycle urbain comme moyen de déplacement), comme ses ruptures (la chasse dans le partage de la Nature) et ses évolutions (le ruban et le genre). Cette nouvelle inflexion prend son sens, non seulement dans la réflexion sur un changement muséographique, mais aussi dans la politique d'acquisition et la structuration des équipes scientifique et de médiation qui doivent intégrer systématiquement l'analyse du fait sociétal dans leur discours d'experts techniques. 
Fig.7. Rallye Audax du Mans en 1939

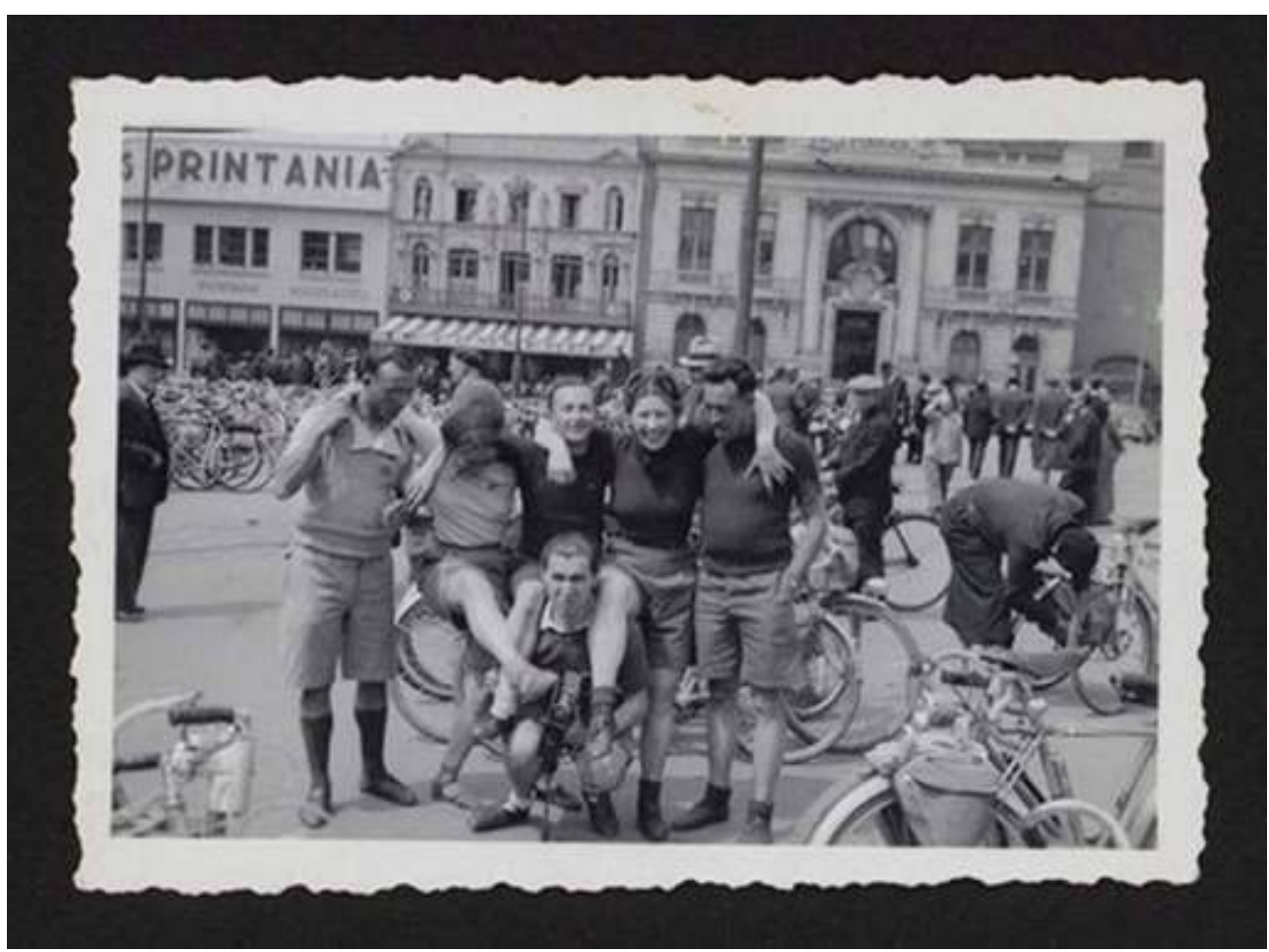

Cette photographie illustre la pratique du cyclotourisme et ses liens avec les premiers congés payés. C'est un exemple de contextualisation de l'objet technique dans une évolution sociétale puisque c'est l'invention du dérailleur, donc la démultiplication qui va permettre de réduire l'effort dans les montées, participant au développement du cyclotourisme. A la fin des années 1930, le cyclotourisme fait partie des mouvements pour une forme de vie plus saine, au grand air, avec des orientations naturiste et végétarienne.

Documentation cycle - musée d'Art et d'Industrie

\section{Conclusion}

Changer de paradigme s'inscrit dans la durée car la mutation doit innerver l'ensemble des acteurs de l'institution muséale. Le nouveau projet culturel et scientifique du musée d'Art et d'Industrie couvre dix années pour donner le temps de porter ce changement dans un contexte de forte évolution de la stratégie patrimoniale du territoire stéphanois. En effet, Couriot musée de la Mine, le musée d'Art et d'Industrie et le musée du Vieux Saint-Étienne, dont les collections ont été transférées à la ville en 2018, sont portés dans le même document cadre dans lequel l'objet technique occupe une place fondamentale. 


\section{BIBLIOGRAPHIE}

BARGUES Cécile, Maurice Allemand ou comment l'art moderne vint à Saint-Étienne (1947-1966) une histoire des collections, Saint-Étienne, Fabelio, 2021

BESSE Nadine, « Aux origines de la collection », in COLLECTIF, Armes de chasse de la mèche à la percussion sur capsules, Collection musée d'Art et d'Industrie de Saint-Étienne, Paris, SaintÉtienne, Somogy, musée d'Art et d'Industrie, 2005

FLICHY Patrice, « Technique, usage et représentations », Réseaux, 2(2-3), 2008, p.147-174

MAIROT Philippe, « Musée et technique », Terrain, n¹6, 1991, p.131-138 ; https://doi.org/ 10.4000/terrain.3004

PASSINI Michela, «VACHON, Marius », in COLLECTIF, Dictionnaire critique des historiens de l'art actifs en France de la Révolution à la Première Guerre Mondiale, publication numérique de l'Institut National d'Histoire de l'Art, 2014

VACHON Marius, Rapports à M. Edmond Truquet, Sous-Secrétaire d'État, sur les musées et les écoles d'art industriel et sur la situation des industries artistiques en Allemagne, Autriche-Hongrie, Italie et Russie,

Paris, A. Quantin, 1885

\section{RÉSUMÉS}

Le musée d'Art et d'Industrie de Saint-Étienne est, dès sa fondation dans les années 1820 , voué à l'objet technique. Les collections d'armes, de rubans et de cycles sont considérées parmi les plus importantes en Europe. Si les collections doivent servir de modèles aux industries, c'est sous l'impulsion de Marius Vachon à la fin du XIX ${ }^{\mathrm{e}}$ siècle qu'elles sont véritablement érigées en modèles. La valeur didactique de ces objets techniques perdure jusqu'à l'ouverture au public d'un musée rénové en 2001. La valeur de l'objet technique glisse de la pédagogie à la valeur d'expert, ce phénomène étant accentué par la perte de lien avec la fabrication sur le territoire. Le projet de 2021 consiste à recréer des liens entre le public et l'objet technique sur des valeurs en constante évolution.

Since its foundation in the 1820s, the Saint-Étienne Museum of Art and Industry has been devoted to technical matters. The collections of weapons, ribbons and cycles are considered among the most important in Europe. If the collections were to serve as models for industries, it was at the instigation of Marius Vachon at the end of the 19th century that they were truly set up as models. The didactic value of these technical objects lasted until the opening to the public of a renovated museum in 2001. The value of the technical object slipped from pedagogy to expert value, this phenomenon being accentuated by the loss of link with manufacturing in the territory. The 2021 project consists of recreating links between the public and the technical object on constantly evolving values.

\section{INDEX}

Mots-clés : histoire des techniques, muséographie, musée, objet technique Keywords : history of technology, museography, museum, technical object 


\section{AUTEUR}

\section{MARIE-CAROLINE JANAND}

Docteur en Histoire de l'Art, je dirige depuis fin 2017 le musée d'Art et d'Industrie et Couriot musée de la Mine. Ces deux musées de la ville de Saint-Étienne portent l'appellation Musée de France. Les axes de la recherche, tournés vers les industries du territoire ont été modifiés dans le cadre du document stratégique qu'est le nouveau Projet Scientifique et Culturel, dorénavant commun aux deux institutions. C'est ainsi que les musées interviennent dans le champ social autour des spécificités des objets techniques conservés. Dans ce cadre, je suis commissaire de nombreuses expositions temporaires sur des sujets variés ( $«$ Vendre de tout, être partout. Casino », « Kata, catastrophes minières » ...) et rédactrice ou directrice de rédaction de plusieurs ouvrages liés à ces expositions. Avant cela, je me suis attachée comme expert en estampes anciennes à valoriser les fonds régionaux d'estampes et de dessins, publiant articles et catalogues d'exposition. 